\title{
An experimental evaluation of localization methods used in wireless sensor networks
}

\author{
Mostapha Laaouafy, Fatima Lakrami, Ouidad Labouidya, Najib Elkamoun \\ Departement of Physics, Laboratory of Sciences and Technologies of Information and Communication, Faculty of Science, Chouaib \\ Doukkali University, El Jadida, Morocco
}

\begin{tabular}{l}
\hline \hline Article Info \\
\hline Article history: \\
Received Apr 24, 2021 \\
Revised Dec 22, 2021 \\
Accepted Jan 10, 2022 \\
\hline
\end{tabular}

\section{Keywords:}

Centroid

Localization

Localization accuracy

MinMax

Multilateration

Trilateration

WSN

\begin{abstract}
The problem of localization in wireless sensor networks has received considerable attention from researchers over the past decades. Several methods and algorithms have been proposed to solve this problem. The effectiveness of these algorithms depends on the accuracy of the estimated positions and the information required to calculate the coordinates. In this paper, we propose to evaluate four of the most commonly used localization methods in sensor networks. Our study considers a mathematical description of the studied methods in order to evaluate their complexity, and then a practical implementation on the simulation tool Cooja. We evaluate the performance of the studied methods as a function of the number of deployed sensor nodes and their degree of mobility in terms of several performance metrics. The objective is to reveal the most suitable localization method for a particular case of deployment. Improvement proposals are also provided to improve the most relevant localization method for the investigated study.
\end{abstract}

This is an open access article under the CC BY-SA license.

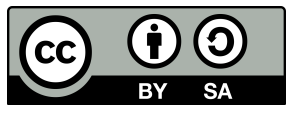

\section{Corresponding Author:}

Mostapha Laaouafy

Departement of Physics, Laboratory of Sciences and Technologies of Information and Communication

Faculty of Science, Chouaib Doukkali University

Jabran Khalil Jabran Avenue, B.P 299-24000, El Jadida, Morocco

Email: mostapha.laaouafy@gmail.com

\section{INTRODUCTION}

With the proliferation of smart objects, localization has become a critical component in deploying future IT services and applications. These future applications will mainly involve the exchange of time-sensitive, fresh and regular information for monitoring and control, as in the case of autonomous vehicles [1]. Although many localization methods have emerged for ad hoc networks, few of them can adapt to all types of environments and support the multiple constraints of wireless communications, most notably wireless sensor networks. Wireless sensor network (WSN) have become increasingly popular in recent years and have attracted a great deal of interest from researchers due to their wide range of applications. Many applications rely on the knowledge of the location of sensor nodes. An event detected by a sensor is only useful in such applications if information about its geographical location is provided. This type of deployment requires calculating sensor positions in a fixed coordinate system, hence the need for localization algorithms. Indeed, the localization of nodes is an essential task in deploying a sensor network to locate the various events occurring in the monitored area and develop protocols for routing the collected information, and data aggregation.

The performance of a localization method depends mainly on its accuracy but also on energy and power consumption. Sensor networks present particular physical and transmission constraints [2], which com- 
plicates the development of a generic localization method regardless of the deployed application. Locating mobile nodes in a sensor network consists of determining these nodes' positions autonomously and periodically without using a fixed infrastructure. Some applications rely mainly on the detection and reporting of the events, which require the knowledge of the exact coordinates of nodes detecting the event. Localization is also necessary for close-range applications that allow different users who are physically close to each other to share some of their information and locate available data. The importance of localization in sensor networks also reveals itself in the management of certain functionalities specific to sensor networks, such as geographic routing.

To help network designers in determining which techniques are appropriate for their applications, authors in [3] present a classification to compare different localization techniques. This classification is based on several key features like the presence of anchor(s), implementation manner, range measurements, and infrastructure type. Chelouah et al. [4] provides a detailed classification of many algorithms of localization in mobile WSNs (MWSNs). Localization techniques, anchor-based/cooperative, network mobility, and information state are all factors that go into the classification. Shieh et al. [5] addresses the localization problem using heuristic optimization approaches, while Darakeha et al. [6] proposes a distributed Range-Free localization algorithm called distributed cooperative and range-free localization algorithm for WSNs (DCRL-WSN), which offers high accuracy. For both approaches, the authors propose distributed algorithms, which means that the sensor nodes are responsible for processing and executing these algorithms, this can increase the cost of computation and subsequently the power consumption, leading to a rapid decrease in network lifetime, especially in hostile regions. Zhang et al. [7] propose a three-dimensional localization algorithm that combines received signal strength indicator (RSSI) and time of arrival (TOA) ranging information, as well as a single movable anchor node to determine the precise distance between the unknown node and the anchor node. The maximumlikelihood estimation method based on obtained ranging values is used to estimate the position of unknown nodes. Simulation results show that the proposed algorithm had lower localization energy consumption and higher localization accuracy, but it requires a large computing capacity.

Zhang and $\mathrm{Wu}[8]$ develop a localization algorithm that allows estimating the positions of several sources in a three-dimensional space using direction-of-arrival (DOA); the results of the simulations show that the proposed method could reduce the computational cost without compromising the accuracy of the estimate. Ibrahim et al. [9] suggested a new range-based localization algorithm called triple mobile anchors for localization (TMAL). This technique is based on three mobile sensors that come together to create a moving triangle capable of locating unknown sensor nodes using received signal strength indicator (RSSI). The simulation results show that this algorithm gives good accuracy. However, authors did not consider energy consumption since they assume that the batteries can be charged to avoid their depletion.

In this paper, we propose a mathematical modeling of four localization methods for sensor networks. We also conduct a comparative study by simulation of the four investigated methods using the Cooja simulator. Our objective is to evaluate the deployment limits of the evaluated methods in the face of the increase in the number of nodes and the network's mobility. Our work is intended as a perspective for the improvement/development of a simple and reliable localization algorithm while considering sensor networks' limits and constraints. The rest of the paper is organized as follows: Section 2 describes the research method. Section 3 presents the simulation and results, while section 4 concludes the paper.

\section{RESEARCH METHOD}

Monitoring/controlling an area of interest is one of the principal purposes of wireless sensor networks (WSN) [10]. The anchors are particular nodes of witch positions are known and allow to build a complete network mapping, which is required because a measurement reflects the state of a specific point. Localization algorithm, measurement technologies and position calculation are the three parts that build a localization system [11]. When there is no knowledge about the location of a wireless sensor network's elements in the deployment environment, the collected data may become of limited usefulness. For any type of processing operation, it is necessary to estimate the location of these sensors at any given moment and with a high accuracy. This can be performed on the basis of the assumed known position of anchors and an inter-sensor range measurements such as received signal strength indication (RSSI) [12]. The localization problem is still one of the important subjects of many research in different fields. In the following paragraphs, we present a description of four methods of localization most deployed by researchers in the field, which are: Trilateration [13], Centroid [14], 
MinMax [15] and Multilateration [16]. These methods have the advantage of being independent of satellites and GPS-based geolocation systems. They are of reduced complexity and offer high accuracy. This makes them highly recommended for embedded systems with limited battery capacity such as wireless sensor networks.

\subsection{Trilateration}

This method is based on the known distances between the target and several anchors as well as its spatial coordinates. Consider a network with three anchors $B_{1}\left(x_{1}, y_{1}\right), B_{2}\left(x_{2}, y_{2}\right)$ and $B_{3}\left(x_{3}, y_{3}\right)$ and a mobile node $\mathrm{M}(\mathrm{x}, \mathrm{y})$ that needs to identified their coordinates [13]. To begin, the received signal strength indicator (RSSI) approach must be used to determine the distances between the mobile node and the three anchors. The signal strength depends on distance and transmitting Power value and then can be deployed to calculate the distance between two sensors. So, the RSSI [17] technique estimates the distance between a transmitter and a receiver based on the the received signal power of a giving data/control packet. In free space, the general formula for calculating RSSI is:

$$
P_{r}=P_{r 0}-20 \log _{10}\left(\frac{d}{d_{0}}\right)
$$

with:

- $d$ : is the difference in distance between the transmitter and receiver.

- $d_{0}$ : is the user-specified distance.

- $P_{r 0}$ : is the signal strength estimated from the transmission rate at the start.

The data transfers allowed the mobile to know anchor positions and the triplet $(D 1, D 2, D 3)$ has been produced by executing the distance measurement protocol. The mobile node can estimate its position by using (2) and (3) as a guide.

$$
\begin{aligned}
& \left(X-X_{1}\right)^{2}+\left(Y-Y_{1}\right)^{2}=D 1^{2} \\
& \left(X-X_{2}\right)^{2}+\left(Y-Y_{2}\right)^{2}=D 2^{2}
\end{aligned}
$$

As shown in Figure 1, the sought position is the point where the circles $C 1(B 1 ; D 1)$ and $C 2(B 2 ; D 2)$ cross. In the general scenario, $C 1$ and $C 2$ intersect at $M$ and $M^{\prime}$. Thanks to the anchor node $B 3$, the mobile node position is one of these two points.

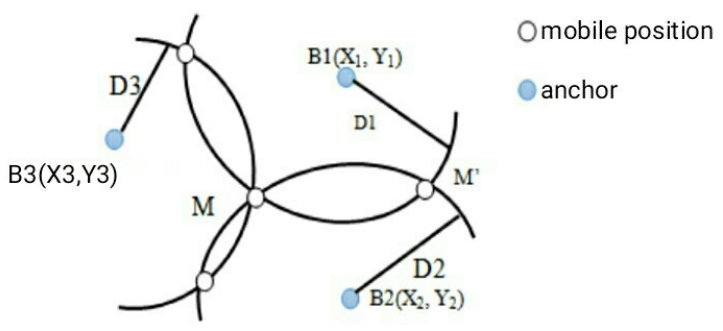

Figure 1. Trilateration principle [18]

\subsection{Centroid}

As shown in Figure 2, the centroid is the point at where the triangle's three medians cross. The triangle's gravity center can be determined by taking the average of the $\mathrm{X}$ and $\mathrm{Y}$ coordinates of all triangle vertices. The centroid localization method relies on a thick layer of references, with each mobile node receiving notification from a few beacons [14]. By determining the center position of all received anchor nodes depending on the assumption of round radio propagation, every mobile node can estimate its location. The centroid localization mechanism requires no cooperation between reference nodes and provides a decent level of localization accuracy. All anchors must communicate their coordinates to all mobile nodes within their transmission area to execute the centroid algorithm, and all mobile nodes must compute their location $M(x, y)$ using (4).

$$
M(x, y)=\left(\frac{x_{1}+x_{2}+x_{3}}{3}, \frac{y_{1}+y_{2}+y_{3}}{3}\right)
$$




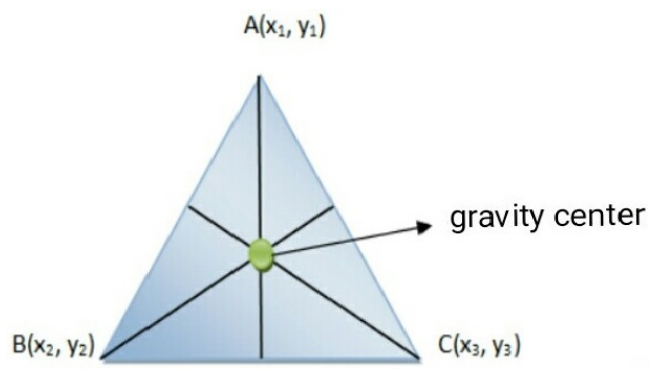

Figure 2. Centroid principle [18]

\subsection{MinMax}

This method's principle consists of associating for each anchor a container area with the sensor node to be located, as shown in Figure 3 . These areas are constructed as (5) [15].

$$
\left(X_{i}-d x_{i} ; Y_{i}-d y_{i}\right) \quad \text { and } \quad\left(X_{i}+d x_{i} ; Y_{i}+d y_{i}\right) ; \quad i=A, B, C
$$

The intersection of these areas forms a new zone defined by (6).

$$
\left(\max \left(X_{i}-d x_{i}\right) ; \max \left(Y_{i}-d y_{i}\right)\right) \quad \text { and } \quad\left(\min \left(X_{i}+d x_{i}\right) ; \min \left(Y_{i}+d y_{i}\right)\right) ; \quad i=A, B, C
$$

The sensor node $\mathrm{M}$ to be located estimates its position as the center of gravity of this area using (7).

$$
(X ; Y)=\left(\frac{\max \left(X_{i}-d x_{i}\right)+\min \left(X_{i}+d x_{i}\right)}{2} ; \frac{\max \left(Y_{i}-d y_{i}\right)+\min \left(Y_{i}+d y_{i}\right)}{2}\right) ; \quad i=A, B, C
$$

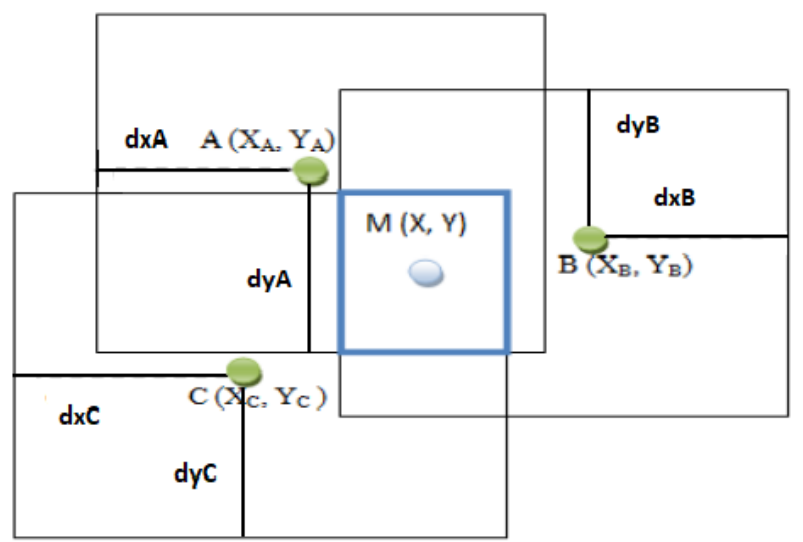

Figure 3. MinMax principle

\subsection{Multilateration}

It is an extension of trilateration [14] that uses more than three anchors to locate sensor nodes. Multilateration minimizes the error margin due to the high number of anchors. The multilateration scheme is illustrated in Figure 4. The estimation of the position of the sensor node $\mathrm{S}$ using multilateration results from the solution of (8).

$$
\left(x-x_{A i}\right)^{2}+\left(y-y_{A i}\right)^{2}=d_{i}^{2}
$$

$\left(x_{A i} ; y_{A i}\right)$ are the coordinates of the anchors $A_{i}$ whatever $i=1 . . n(n>3)$ while $(x ; y)$ are the coordinates of the sensor node $\mathrm{S}$ to be computed. 


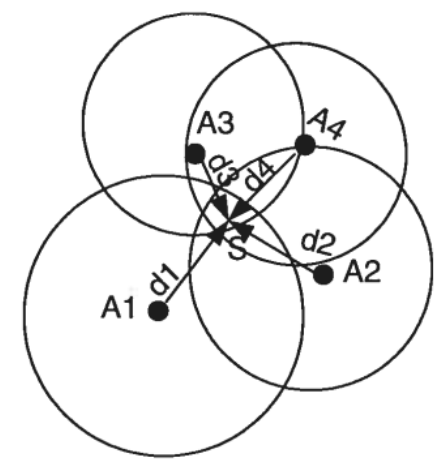

Figure 4. Multilateration principle [19]

\section{SIMULATION AND RESULTS}

There are few studies on modeling and simulating localization methods in wireless sensor networks. Authors focus generally on geolocalization methods, where at least one terminal is capable of being located using a satellite positioning system and a GPS receiver. These methods are known for their high precision error. This section provides a description of some examples of related works. Sheltami et al. [20] proposed that three known localization protocols (fingerprint, centroid, and DV-Hop) are evaluated in terms of accuracy and power consumption; simulation results show that fingerprint is very accurate than centroid and DV-Hop, but the latest outperform in terms of power consumption and stability.

Grigulo and Becker [21] focused on validating experimentally the technique named efficient geometrybased localization (EGL). This technique locates static sensor nodes in an experimental field with an efficient, distributed, and scalable manner. A unmanned aerial vehicles (UAV) system with autonomous flight and a lowcost global navigation satellite system (GNSS) receiver will carry the mobile sink node. The EGL technique was validated by experimental results comparing localization with real time kinematic (RTK) and standalone GNSS technique. Priya and Ali [22] modeled and simulated the localization problem in WSN using an improved DV-Distance algorithm combined with trilateration method to prevent increasing localization error. The results show that the estimated and the exact coordinates are very close.

In a previous work [18], we conducted a comparative study in terms of precision and energy consumption of the most well known and free localization methods that are: trilateration and centroid methods. The current work presents an extension of the previous study by simulating 4 localization methods: trilateration, centroid, MinMax and reduced MinMax (MinMax method with a minimal number of anchors). We also provide some perspectives for the enhancement of the localization method that manifests the best results.

\subsection{Simulation platform}

The COOJA simulator stands for COntiki Os Java simulator. It's a simulator for sensor networks. The Contiki OS is a portable operating system developed for devices with limited resources, such as sensor nodes [23], [24]. Thanks to this simulator, we can efficiently test a code written in C language without using real flash sensors. We can allocate any number of nodes over a given area. We then visualize in real-time (or accelerated) the evolution of the network topology. In a simulation, we have several windows like shown in Figure 5 .

- The network window displays the network's graphical representation and shows us all nodes in the simulated network.

- The simulation control window is where the simulation is started, paused, stopped and wholly reloaded.

- The notes window is where we can put notes for our simulation.

- The mote output window is where the sensor outputs are printed. A text field allows us to enter a filter to target a particular sensor or message type.

- The timeline window displays all communication events in the simulation over time and very convenient to understand what is happening in the network.

The mobility model describes the movement pattern of mobile nodes and how their localizations change in term of speed and frequency. This model defines also the trajectory of mobility. In this work, we use The random mobility model where mobile nodes that change their positions randomly and periodically. 


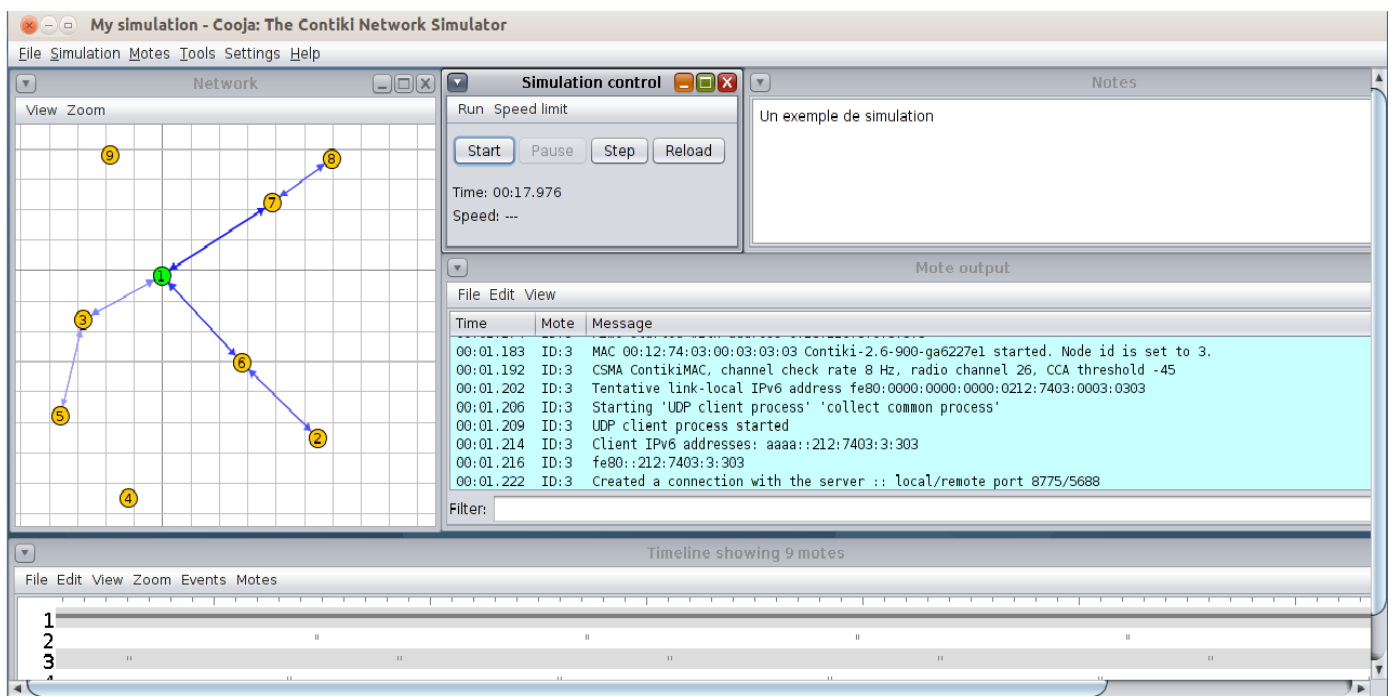

Figure 5. Cooja simulator graphical interface

\subsection{Localization accuracy}

The localization accuracy [25] is evaluated by analyzing the error in the derived localization. A localization method is more accurate when its error is smaller. The estimated error between the mobile node's exact and estimated position is determined using (9).

$$
\text { Error }=\sqrt{\left(x_{\text {exact }}-x_{\text {estim }}\right)^{2}+\left(y_{\text {exact }}-y_{\text {estim }}\right)^{2}}
$$

\subsection{Trilateration implementation}

The network topology presenting the simulation scenario contains 14 sensor nodes deployed in an area of $90 \times 90 \mathrm{~m}^{2}$, including eight mobile devices and six anchor devices. $40 \mathrm{~m}$ is the wireless communication range, and the transmission rate is $40 \%$. The mobile node starts executing the algorithm of the trilateration method by broadcasting a Hello message within its transmission range to all anchors. The mobile node measures the RSSI value for each beacon when a neighbor node responds to the Hello message. The distance between mobile nodes and neighbor anchors is calculated using (1). By resolving the equation system created by (2) and (3), we obtain the mobile node position. The (10) and (11) are the equation system's solutions using Python programming language. Figure 6 represents the simulation results and the error between the exact and estimated positions in the Trilateration localization method's.

$$
\begin{gathered}
x=-\frac{1}{2\left(x_{1}-x_{2}\right) \times\left(x_{1}^{2}-2 x_{1} x_{2}+x_{2}^{2}+y_{1}^{2}-2 y_{1} y_{2}+y_{2}^{2}\right)}\left(y_{1}-y_{2}\right) \times\left(-D_{1}^{2} y_{1}+D_{1}^{2} y_{2}+D_{2}^{2} y_{1}\right. \\
-D_{2}^{2} y_{2}+x_{1}^{2} y_{1}+x_{1}^{2} y_{2}-2 x_{1} x_{2} y_{1}-2 x_{1} x_{2} y_{2}+x_{2}^{2} y_{1}+x_{2}^{2} y_{2}+y_{1}^{3}-y_{1}^{2} y_{2}-y_{1} y_{2}^{2}+y_{2}^{3} \\
-\sqrt{\left(-D_{1}^{2}+2 D_{1} D_{2}-D_{2}^{2}+x_{1}^{2}-2 x_{1} x_{2}+x_{2}^{2}+y_{1}^{2}-2 y_{1} y_{2}+y_{2}^{2}\right)} \times\left(x_{1}-x_{2}\right) \\
\times \sqrt{\left(D_{1}^{2}+2 D_{1} D_{2}+D_{2}^{2}-x_{1}^{2}+2 x_{1} x_{2}-x_{2}^{2}-y_{1}^{2}+2 y_{1} y_{2}-y_{2}^{2}\right)} \\
\left.+\left(D_{1}^{2}-D_{2}^{2}-x_{1}^{2}+x_{2}^{2}-y_{1}^{2}+y_{2}^{2}\right) \times\left(x_{1}^{2}-2 x_{1} x_{2}+x_{2}^{2}+y_{1}^{2}-2 y_{1} y_{2}+y_{2}^{2}\right)\right) \\
y=\frac{1}{2\left(x_{1}^{2}-2 x_{1} x_{2}+x_{2}^{2}+y_{1}^{2}-2 y_{1} y_{2}+y_{2}^{2}\right)} \times\left(-D_{1}^{2} y_{1}+D_{1}^{2} y_{2}+D_{2}^{2} y_{1}\right. \\
-D_{2}^{2} y_{2}+x_{1}^{2} y_{1}+x_{1}^{2} y_{2}-2 x_{1} x_{2} y_{1}-2 x_{1} x_{2} y_{2}+x_{2}^{2} y_{1}+x_{2}^{2} y_{2}+y_{1}^{3}-y_{1}^{2} y_{2}-y_{1} y_{2}^{2}+y_{2}^{3} \\
+\sqrt{\left(-D_{1}^{2}+2 D_{1} D_{2}-D_{2}^{2}+x_{1}^{2}-2 x_{1} x_{2}+x_{2}^{2}+y_{1}^{2}-2 y_{1} y_{2}+y_{2}^{2}\right)} \times\left(-x_{1}+x_{2}\right) \\
\times \sqrt{\left.\left(D_{1}^{2}+2 D_{1} D_{2}+D_{2}^{2}-x_{1}^{2}+2 x_{1} x_{2}-x_{2}^{2}-y_{1}^{2}+2 y_{1} y_{2}-y_{2}^{2}\right)\right)}
\end{gathered}
$$




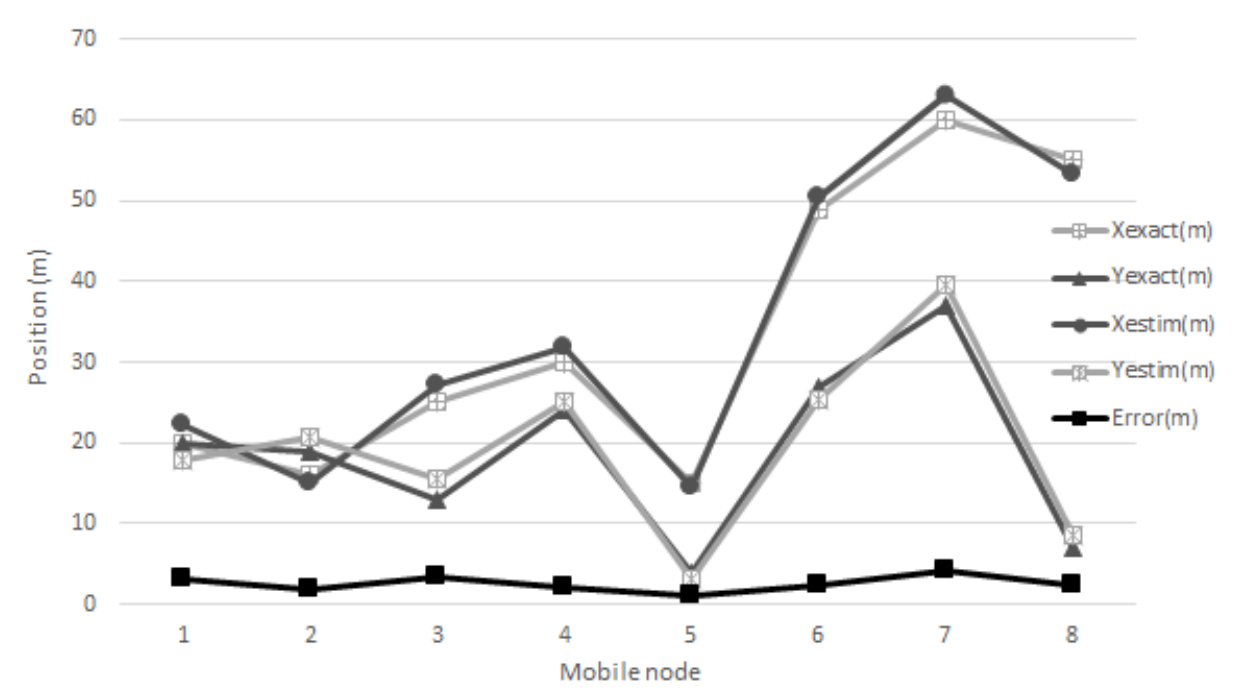

Figure 6. Trilateration localization method results

\subsection{Centroid implementation}

This method's experiments are carried out by deploying in an area of $90 \times 90 \mathrm{~m}^{2}$ in the Network window of Cooja simulator 16 sensor nodes, six of which are anchor devices and 10 of which are mobile devices. The wireless communication range is $40 \mathrm{~m}$, and the transmission rate is $40 \%$. When we click on the button start in the simulation control window, the network starts to communicate. The mobile node gets data from the first three beacons and uses (4) to compute its own position. Figure 7 represents the results of simulations and the precision error relative to the centroid method.

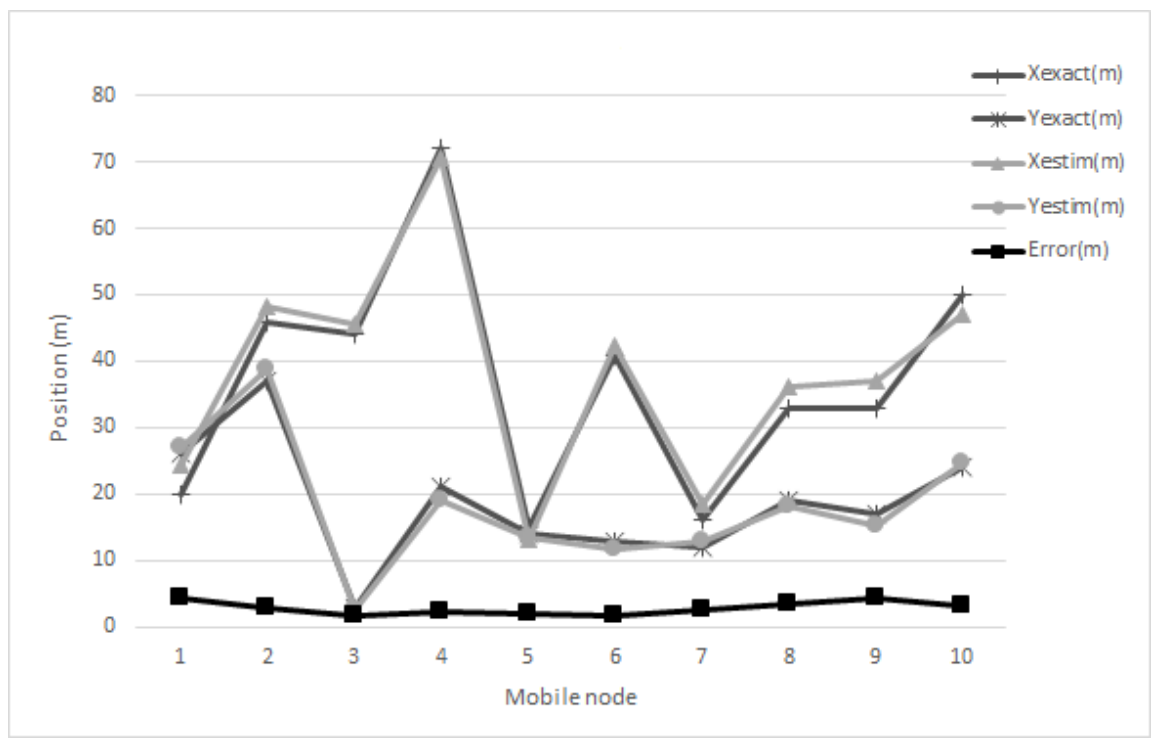

Figure 7. Centroid localization method results

\subsection{MinMax implementation}

To simulate this method, we first configure a topology that contains 18 sensor nodes, eight of which are anchor devices and 10 of which are mobile devices. The topology is represented in the Network window of the Cooja simulator by a $90 \times 90 \mathrm{~m}^{2}$ area with a wireless communication range of $40 \mathrm{~m}$ and a transmission 
rate of $40 \%$. When the start button is pressed, the mobile nodes begin exchanging data with their neighboring anchors. Figure 8 shows the simulation results and the precision error for the MinMax method.

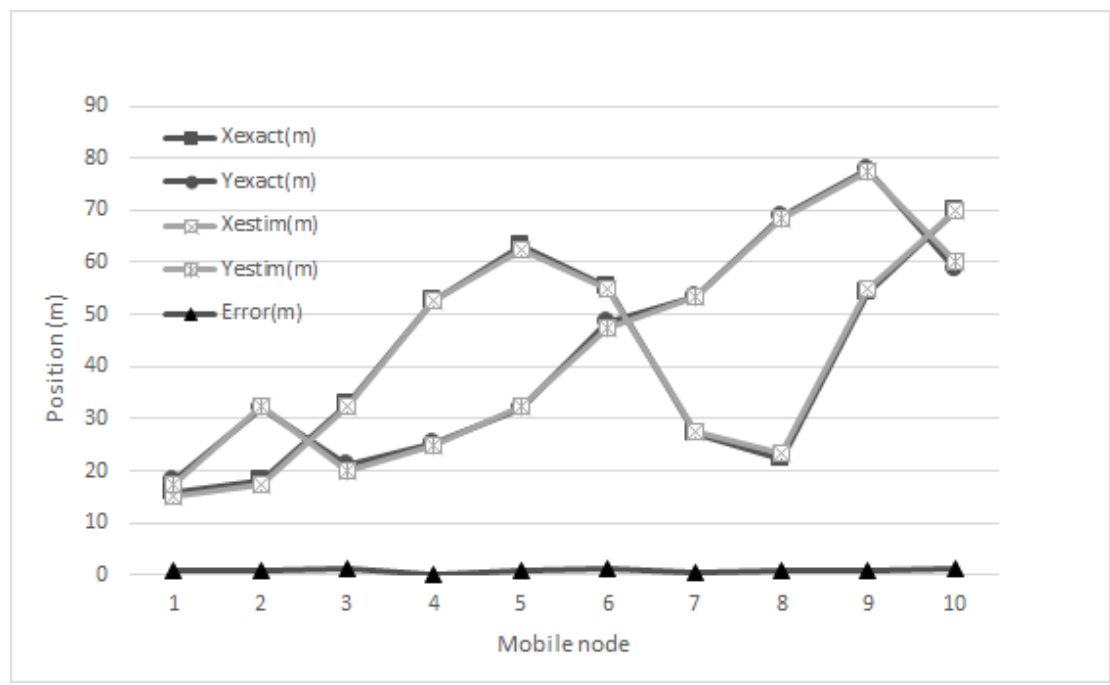

Figure 8. MinMax localization method results

\subsection{Reduced MinMax implementation}

To simulate this method, we adopt the same sensor nodes topology used to evaluate the MinMax method, but this time we configure only three anchor nodes while the other 15 nodes are set as mobile nodes dispatched in an area of $90 \times 90 \mathrm{~m}^{2}$ with a transmission rate of $40 \%$ and a communication range of $100 \mathrm{~m}$. In this method, the same evaluation principle of the original MinMax method is replicated but with a reduced number of anchor nodes and an increased transmission range. Figure 9 shows the simulation results and the precision error obtained for this method.

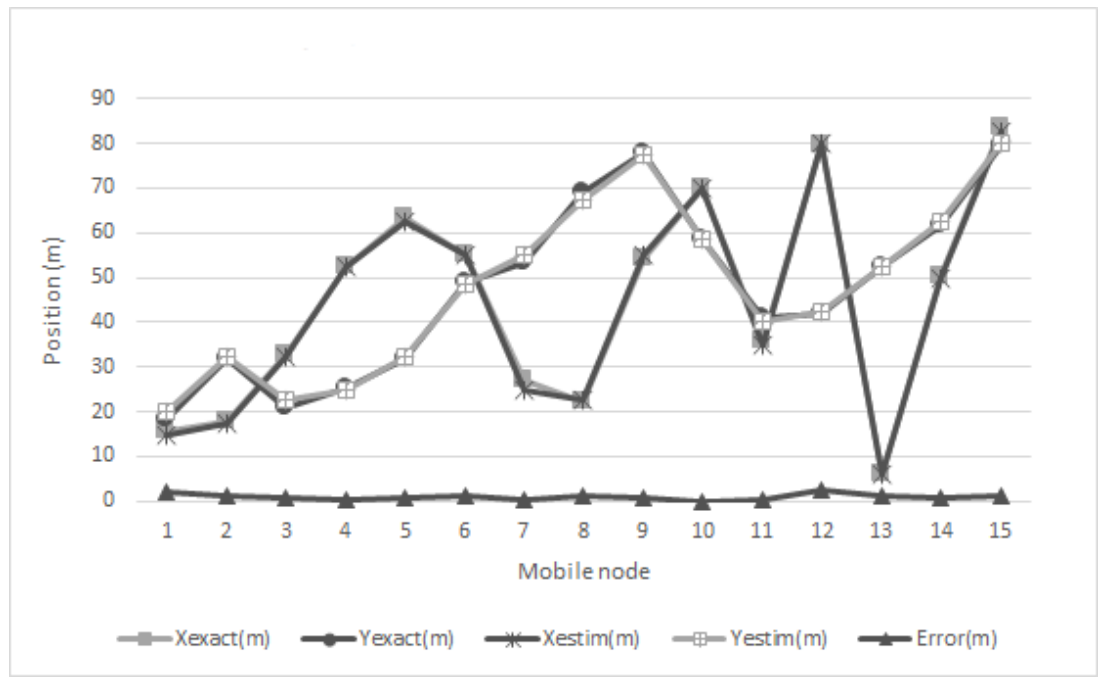

Figure 9. Reduced MinMax localization method results

\subsection{Discussion of results}

To compare the different simulated methods, we will refer to their accuracy in term of precision errors. In Figure 10 it is clear that the localization error of trilateration and centroid methods exceeds four meters while it does not exceeds one and a half meters for the MinMax method. Therefore we can deduce that the MinMax 
method is more precise than both trilateration and centroid methods, while the reduced MinMAx method allows locating many mobile sensor nodes with better accuracy using a reduced number of anchors.

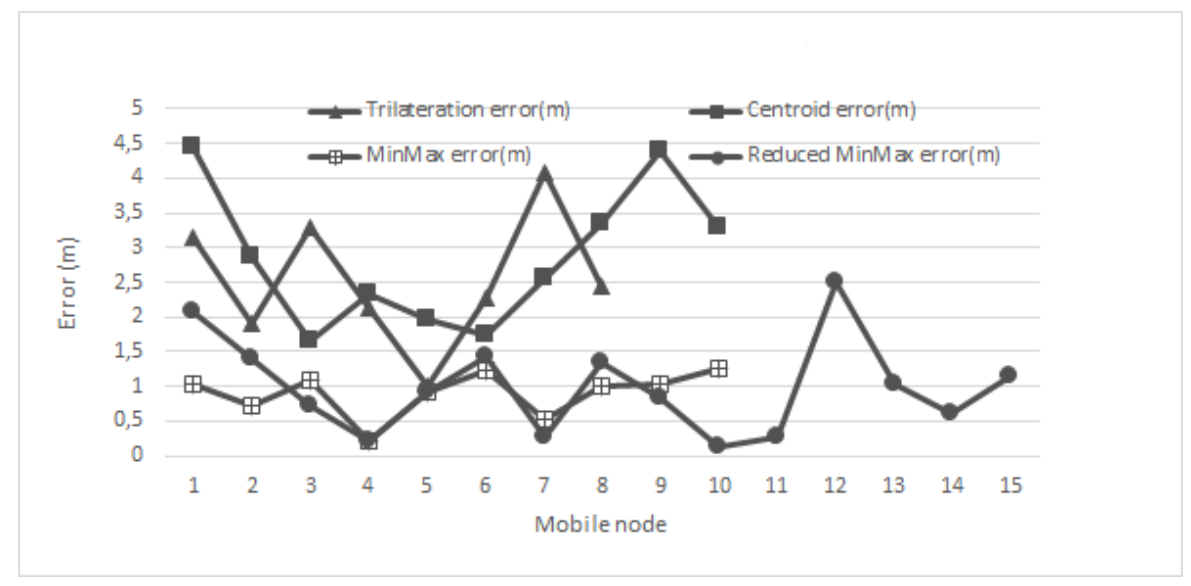

Figure 10. Localization methods error comparison

In terms of energy consumption the trilateration method is the most consuming because it uses the RSSI technique to estimate the distance between anchors and the mobile node before running its algorithm, and this is not the case for centroid and MinMax methods. To locate every mobile node using MinMax method it is necessary to associate for each anchor a private container area which increases the energy consumption unlike the centroid method where the anchors broadcast their coordinates to every mobile node in their transmission area. So we can conclude like shown in Figure 11 that the centroid method consumes less energy than the trilateration and MinMax methods.

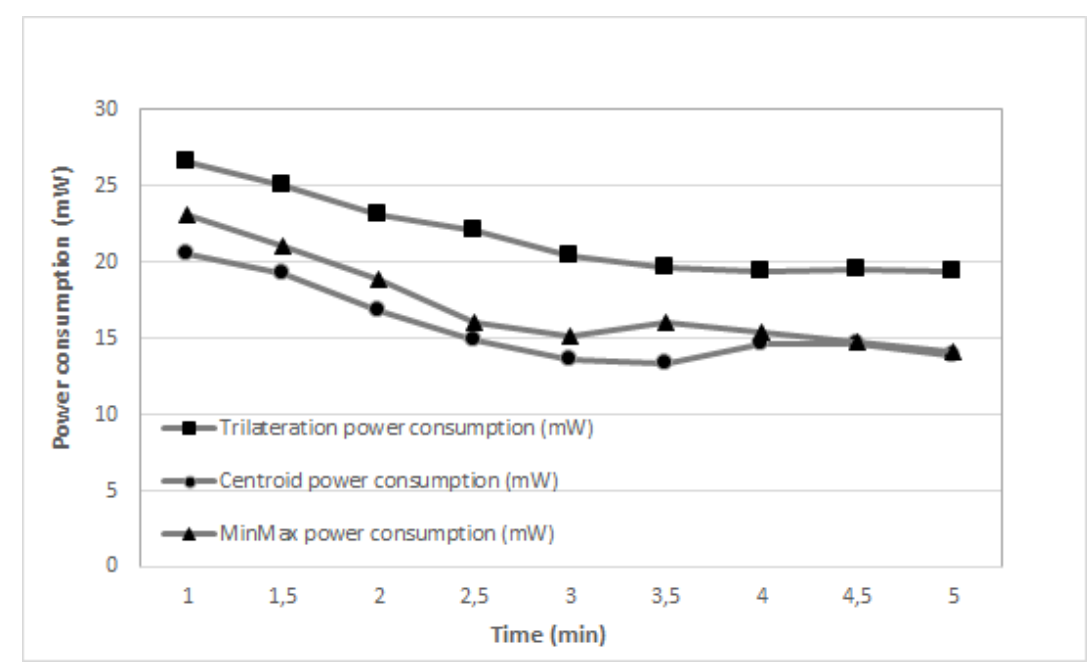

Figure 11. Localization methods power consumption

\section{CONCLUSION}

We presented in this paper a mathematical modeling and a comparative study throught simulation of four basic localization methods: trilateration, centroid, MinMax and reduced MinMax. Simulation results show that the MinMax method is more accurate thand other methods, while the centroid method is concluded to be the best in terms of energy consumption. For the centroid and trilateration methods; it can be noticed that their performance deteriorates significantly and rapidly as the number of mobile nodes increases. The reduced MinMax gives average but acceptable results in terms of accuracy and energy consumption, its performance 
also degrades when increasing the number of nodes and their degree of mobility, but not as significantly as the other methods. We are currently working on improving the reduced MinMax method to enable its scalability while integrating a better network management especially in the presence of a high number of mobile nodes or anchors.

\section{REFERENCES}

[1] M. A. Uthaib and M. S. Croock, "Vehicle plate localization and extraction based on hough transform and bilinear operations," Indonesian Journal of Electrical Engineering and Computer Science (IJEECS), vol. 20, no. 2, pp. 1088-1097, 2020, doi: 10.11591/ijeecs.v20.i2.pp1088-1097.

[2] J. Zhao, O. Yağan and V. Gligor, "Connectivity in secure wireless sensor networks under transmission constraints," 2014 52nd Annual Allerton Conference on Communication, Control, and Computing (Allerton), 2014, pp. 1294-1301, doi: 10.1109/ALLERTON.2014.7028605.

[3] F. Mekelleche and H. Haffaf, "Classification and comparison of range-based localization techniques in wireless sensor networks," Journal of Communications, vol. 12, no. 4, pp. 221-227, Apr. 2017, doi: 10.12720/jcm.12.4.221-227.

[4] L. Chelouah, F. Semchedine, and L. Bouallouche-Medjkoune, "Localization protocols for mobile wireless sensor networks: A survey," Computers and Electrical Engineering, vol. 71, pp. 733-751, Oct. 2018, doi: 10.1016/j.compeleceng.2017.03.024.

[5] C. -S. Shieh, V. -O. Sai, Y. -C. Lin, T. -F. Lee, T. -T. Nguyen, and Q. -D. Le, "Improved Node Localization for WSN Using Heuristic Optimization Approaches," 2016 International Conference on Networking and Network Applications (NaNA), 2016, pp. 95-98, doi: 10.1109/NaNA.2016.58

[6] F. Darakeh, G.-R. Mohammad-Khani, and P. Azmi, "DCRL-WSN: A distributed cooperative and range-free localization algorithm for WSNs," AEU-International Journal of Electronics and Communications, vol. 93, pp. 289-295, September 2018, doi: 10.1016/j.aeue.2018.05.015.

[7] L. Zhang, Z. Yang, S. Zhang, and H. Yang, "Three-Dimensional Localization Algorithm of WSN Nodes Based on RSSI-TOA and Single Mobile Anchor Node," Journal of Electrical and Computer Engineering, vol. 2019, July 2019, doi: 10.1155/2019/4043106.

[8] Y. Zhang and Y. I. Wu, "Multiple Sources Localization by the WSN Using the Direction-of-Arrivals Classified by the Genetic Algorithm," in IEEE Access, vol. 7, pp. 173626-173635, 2019, doi: 10.1109/ACCESS.2019.2956825.

[9] B. K. Ibrahim, M. A. Mahdi, and M. A. Salman, "Triple Mobile Anchors Approach for Localization in WSN," 2020 International Conference on Computer Science and Software Engineering (CSASE), 2020, pp. 174-179, doi: 10.1109/CSASE48920.2020.9142084.

[10] A. Ali, Y. Ming, S. Chakraborty, and S. Iram, "A Comprehensive Survey on Real-Time Applications of WSN," Future Internet, vol. 9, no. 4, p. 77, November 2017, doi: 10.3390/fi9040077.

[11] A. Boukerche, H. A. B. F. Oliveira, E. F. Nakamura, and A. A. F. Loureiro, "Localization systems for wireless sensor networks," in IEEE Wireless Communications, vol. 14, no. 6, pp. 6-12, December 2007, doi: 10.1109/MWC.2007.4407221.

[12] J. Xu, W. Liu, F. Lang, Y. Zhang, and C. Wang, "Distance Measurement Model Based on RSSI in WSN," Wireless Sensor Network, vol. 2, no. 8, pp. 606-611, August 2010, doi: 10.4236/wsn.2010.28072.

[13] E. Goldoni, A. Savioli, M. Risi, and P. Gamba, "Experimental analysis of RSSI-based indoor localization with IEEE 802.15.4," 2010 European Wireless Conference (EW), 2010, pp. 71-77, doi: 10.1109/EW.2010.5483396.

[14] J. Blumenthal, R. Grossmann, F. Golatowski, and D. Timmermann, "Weighted Centroid Localization in Zigbee-based Sensor Networks," 2007 IEEE International Symposium on Intelligent Signal Processing, 2007, pp. 1-6, doi: 10.1109/WISP.2007.4447528.

[15] H. Will, T. Hillebrandt, Y. Yuan, Z. Yubin, and M. Kyas, "The Membership Degree Min-Max localization algorithm," 2012 Ubiquitous Positioning, Indoor Navigation, and Location Based Service (UPINLBS), 2012, pp. 1-10, doi: 10.1109/UPINLBS.2012.6409781.

[16] L. Asmaa, K. A. Hatim, and M. Abdelaaziz, "Localization algorithms research in wireless sensor network based on Multilateration and Trilateration techniques," 2014 Third IEEE International Colloquium in Information Science and Technology (CIST), 2014, pp. 415-419, doi: 10.1109/CIST.2014.7016656.

[17] R. M. Dellosa, A. C. Fajardo, and R. P. Medina, "Modified fingerprinting localization technique of indoor positioning system based on coordinates," Indonesian Journal of Electrical Engineering and Computer Science (IJEECS), vol. 15, no. 3, pp. 1345-1355, September 2019, doi: 10.11591/ijeecs.v15.i3.pp1345-1355.

[18] M. Laaouafy, F. Lakrami, O. Labouidya, N. Elkamoun, and R. Iqdour, "Comparative study of localization methods in WSN using Cooja simulator," 2019 7th Mediterranean Congress of Telecommunications (CMT), 2019, pp. 1-5, doi: 10.1109/CMT.2019.8931399.

[19] M. boushaba, "Locating nodes in wireless sensor networks," Master of Science thesis, Network Research Laboratory, University of Montreal, Canada, July 2007,

[20] T. R. Sheltami, E. Q. Shahra, and E. M. Shakshuki, "Perfomance comparison of three localization protocols in WSN using Cooja," Journal of Ambient Intelligence and Humanized Computing, vol. 8. no. 3, pp. 373-382, February 2017, doi: 10.1007/s12652-0170451-2.

[21] J. Grigulo and L. B. Becker, "Experimenting Sensor Nodes Localization in WSN with UAV Acting as Mobile Agent," 2018 IEEE 23rd International Conference on Emerging Technologies and Factory Automation (ETFA), 2018, pp. 808-815, doi: 10.1109/ETFA.2018.8502536

[22] S. Priya and A. Ali, "Localization of WSN using IDV and Trilateration Algorithm," Asian Journal of Engineering Technology and Innovation, vol. 4, no. 7, pp. 140-143, May 2016.

[23] F. Osterlind, A. Dunkels, J. Eriksson, N. Finne, and T. Voigt, "Cross-Level Sensor Network Simulation with COOJA," Proceedings. 2006 31st IEEE Conference on Local Computer Networks, 2006, pp. 641-648, doi: 10.1109/LCN.2006.322172.

[24] D. Jabba and P. Acevedo, "ViTool-BC: Visualization Tool Based on Cooja Simulator for WSN," Applied Sciences, vol. 11, no. 16, p. 7665, August 2021, doi: 10.3390/app11167665. 
[25] S. H. Thimmaiah and M. G, "A Range Based Localization Error Minimization Technique for Wireless Sensor Network," Indonesian Journal of Electrical Engineering and Computer Science (IJEECS), vol. 7, no. 2, pp. 395-403, August 2017, doi: 10.11591/ijeecs.v7.i2.pp395-403

\section{BIOGRAPHIES OF AUTHORS}

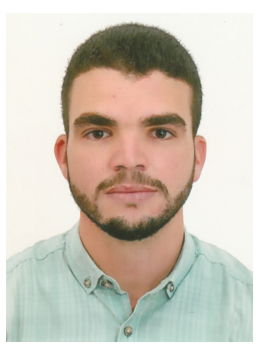

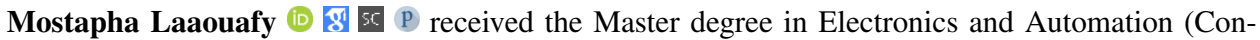
trol, Industrial Computing, Signals and Systems) in 2018 from Cadi Ayyad University, Marrakech, Morocco. Currently, he is a PhD Student at STIC Laboratory, Science Faculty, Chouaîb Doukkali University, El Jadida, Morocco. His main research area is on Wireless Sensor Networks. Exactly, in localization problem in WSN. He can be contacted at email: mostapha.laaouafy@gmail.com.

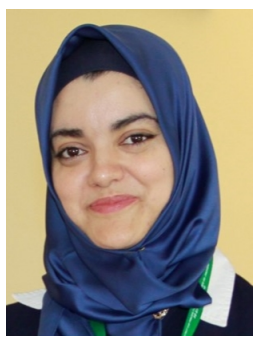

Fatima Lakrami (D) 50 is 5 is a researcher and a professor at Science Faculty, Chouaîb Doukkali University, El Jadida, Morocco. She got her Doctorate in Telecommunication and Networking in 2014 from Chouaîb Doukkali University, El Jadida, Morocco. Her research interests cover wireless networks performance evaluation, VANETs and Security. She can be contacted at email: fatima.lakrami@gmail.com.

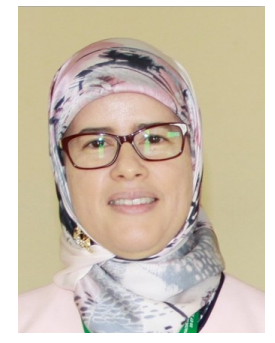

Ouidad Labouidya (1) 56 is currently Professor Researcher at Faculty of Science, University Chouaib Doukkali, El Jadida, Morocco. She obtained her Diploma of an Engineer degree in Electronic Instrumentation and Maintenance in 1992. She received his PHD degree in Science and Technology of Information and Communication from Faculty of Science of University Chouaib Doukkali, Morocco, in 2009. With over 17 years of expertise in ICT, she has conducted several researches and overseas missions in E-learning and Telecommunication Networks. Her research interests include self-training and ICT for education, evaluation in higher education, Computer and Telecommunication Networks. She can be contacted at email: Labouidya.O@ucd.ma.

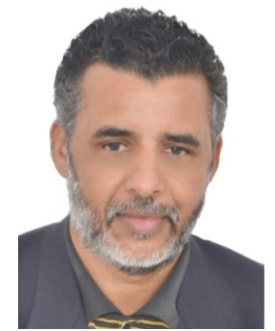

Najib Elkamoun (D) 18 S P is a professor at Faculty of sciences, Chouaib Doukkali University, El Jadida, Morocco. He is a researcher member of STIC laboratory and header of Network and Telecommunications team. His research interest includes: NGN, MPLS, Networks, QoS in mobile networks and wireless networks. He can be contacted at email: Elkamoun@gmail.com. 